\title{
An Algorithm for using Internet of Things (IoTs) to Improve Load Management in Electric Power Grid
}

\author{
Gilbert Mahlangu \\ Cape Peninsula University of \\ Technology, South Africa
}

\author{
Samuel Musungwini \\ Midlands State University, \\ Gweru, Zimbabwe
}

\author{
Sindiso Nleya \\ National University of Science \\ and Technology, Bulawayo \\ Zimbabwe
}

\begin{abstract}
Several new technologies, for example, the Internet of Things (IoTs) continue to surface in the frontage of ubiquitous and pervasive computing. Internet of things is slowly being embraced in different domains to support control and monitoring systems. The advent of microprocessors embedded with communication facilities has enabled the possibility of integrating ICTs within people and their environments. With the power utilities industry grappling with serious issues like load management which has a potential of damaging their equipment, first movers are turning to advanced technologies such as the IoTs to achieve demandside management. The purpose of this study was to conduct a theoretical and empirical review on the approaches used by Power utilities to manage load in order to determine how IoTs can be used particularly by Zimbabwe's Power utility to solve the supply-demand problem. Currently, the load management approaches used by the Power utility in Zimbabwe are load shifting and load shedding. A conceptual model was developed using $\mathrm{C}++$ guided by the algorithm for load management. The model development was motivated by the research gap establish from the literature review and the problem faced by the Power utility in Zimbabwe in balancing demand and supply during peak periods. The idea is modelled around continuous monitoring of the feeder or substation, activating the automatic control and carrying out load allocation procedure based on priority settings, connection, and re-connection timings. The proposed model is also based on the dependence of demand and consumer priority perception. The model will ensure that electricity is available for basic and essential use in times of increased demand. It requires that load demand is constantly monitored to trigger automatic control and appliances should connect to the electric power grid using smart plugs.
\end{abstract}

\section{General Terms}

Microprocessors, embedded, power utility, technologies, demand-side management

\section{Keywords}

Conceptual model, Internet of Things (IoTs), load management, electric, power grid, consumer priority, demand dependence, switchable appliances, Algorithm

\section{INTRODUCTION}

The last few decades have seen several new technologies entering the frontage of ubiquitous and pervasive computing; the most recent one according to [1] being the Internet of Things (IoTs). Internet of things is slowly being embraced in different domains to support control and monitoring systems. The advent of microprocessors embedded with communication facilities has enabled the possibility of integrating ICTs within people and their environments [2]. This integration enables physical objects to be "smart" and intelligent by means of observing their environments, detecting anomalies and communicating with other objects in order to perform a coordinated decision. Its application in various domains has enabled organisations to gain insight into various infrastructure and equipment for decision making.

With the Power utility industry grappling with serious issues like load management which has a potential of damaging their operational equipment and that of customers [3], first movers are turning to advanced technologies such as the IoTs to achieve demand-side management. This technology enables utilities to supply electric power more efficiently and reduce unnecessary blackouts. The platform of IoTs offers Power utilities opportunity to monitor and control electric power grid [4]. Using data from the infrastructure, the Power utility can be alerted to any eventuality and respond expeditiously. Therefore, this platform (IoTs) offers infinite prospects to Power utilities as they can connect their entire infrastructure and personnel to a single communication system.

In this paper, an algorithm which minimizes unnecessary load-shading under high demand of electricity is proposed. This will monitor the amount of load connected to the distribution network, updates the substation/feeder of the Power utility and take actions depending on the result of its measurement and the priority settings. The basic idea is to ensure that the consumption of electric power by devices connected to the distribution network do not exceed the maximum allocation of the substation/feeder. The monitoring of devices should start once the demand of electricity power exceeds $90 \%$ of the maximum allocated in a particular substation/feeder. Once monitoring is activated, devices will be connected or disconnected depending on the priority settings and connectivity time. The algorithm is design in such a way that high priority devices will always be connected to the distribution network.

The paper is organized as follows. Section 2 discusses the background of the study and the motivation. Section 3 describes the vision and the concept of IoTs. Section 4 presents the load management techniques in Power grid in generally and Zimbabwe in particular. Section 5 highlights the role of IoTs in load management. Section 6 gives an overview of the conceptual model and more details about the proposed algorithm for load management. Simulation results are presented and commented in this section. Finally, Section 7 concludes this paper and gives future scope of the study.

\section{BACKGROUND TO THE STUDY}

Load management has become a critical aspect in the distribution and supply chain of electric power, especially to power utilities like Zimbabwe that are faced with capacity constraints in electric power generation [5], [6]. The Power utility in Zimbabwe currently faces critical shortages in electric power generation which has consequently led to the practice of load shedding in order to maintain a balance 
between demand and supply. According to [7], the daily electric power demand is at 2,200 MW against a generating capacity of $770 \mathrm{MW}$ which is not enough to sustain consumer demand. This has left the Power utility with a deficit of 1430 MW.

An analysis of the load management system used by the Power utility in Zimbabwe reveals that the system is programmed to automatically triple the breakers if particular substation or feeder is overstrained by consumer demand. This has resulted in unnecessary blackouts in time of electricity needs. Therefore, it is against this background that the researchers suggest that the Power utility need to incorporate the IoTs technology in order to manage this supply-demand problem.

\section{VISION AND CONCEPT OF INTERNET OF THINGS}

The vision of the IoTs is grounded in the belief that the sound advances in microelectronics, communications and information technology will change the concept of the virtual world [8]. Furthermore, [9] has suggested that the advancements towards the IoTs technology will see the number of connected devices to the internet surpassing the number of connected people. According to [10] and [11], the basic concept of the IoTs describes a system in which physical objects are connected to the internet by sensors to become part of the virtual world. This connectivity reduces human intervention in monitoring and control systems. Therefore, [12] conclude that the ability to react to events in the physical world in an automatic, rapid and informed manner enables a wide variety of business processes to be optimized within the ICT domain.

\subsection{Essential Element and Actions of IoTs}

The devices necessary to implement the technology of IoTs follows under three broad categories [13]: (1) sensors: input devices that detect the environment, (ii) processors: electronic systems that interpret and analyse input data, and (iii) actuators: output devices that respond to processed information by altering the environment via electronic or mechanical means. However, according to [14], for these operations to proceed as required, a number of actions should be performed as indicated in Figure 1 below.

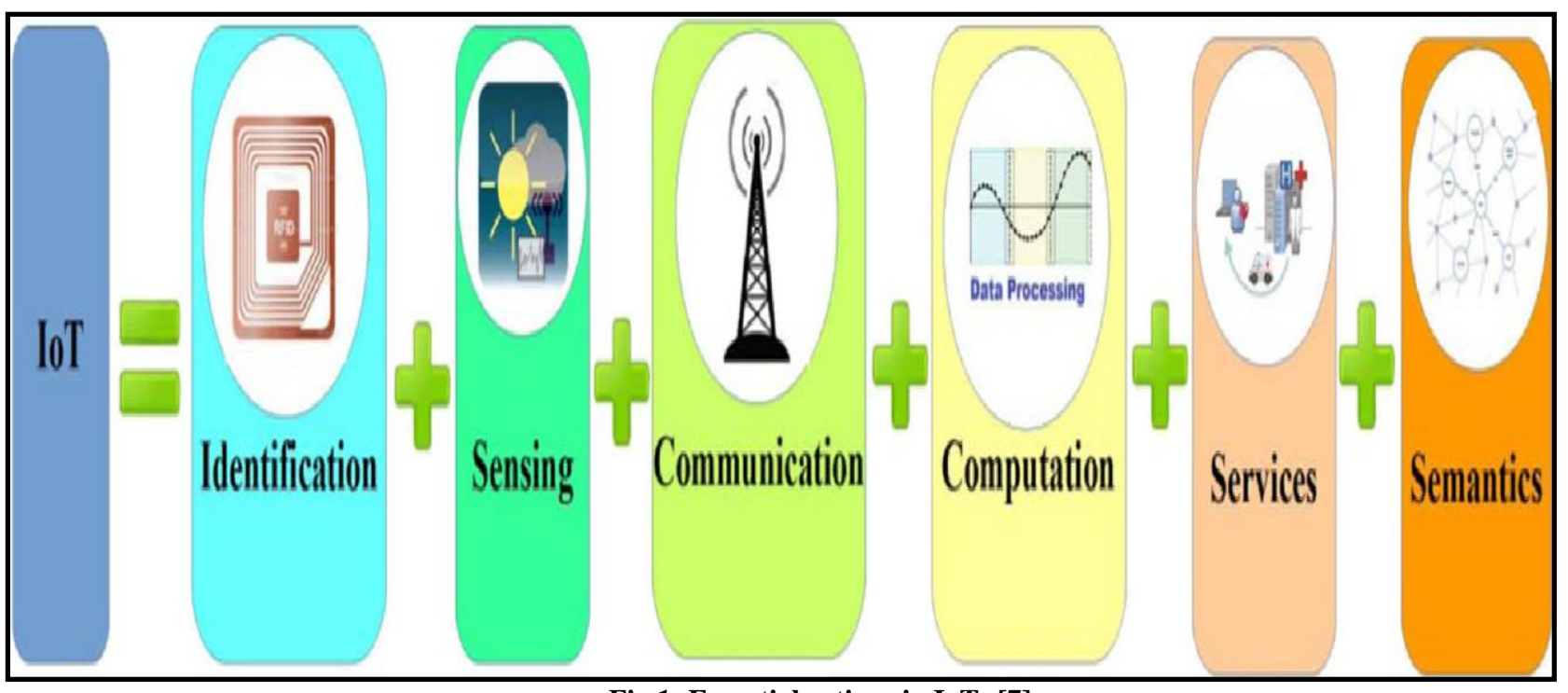

Fig 1: Essential actions in IoTs [7]

\subsubsection{Identification}

The identification process can be seen as the first action to be performed in the IoTs. The action enables IoTs to uniquely name objects on the internet and match their requests with what is known to the system. Objects connected to the internet need to be allocated identification codes so that they can be clearly identified in order to fulfil a matching strategy.

\subsubsection{Sensing}

Sensing in the internet of things refers to the process of acquiring information from related objects with the network [15]. This information is sent to the control centre or other objects so that it can be evaluated against the matching strategy for decision making. It is preferred that the sensing equipment should use low power for long life performance.

\subsubsection{Communication}

For the objects to send acquired information across the network, they should be connected together by specified protocols. These protocols act as enabling technologies sitting in the confluence of objects, sensors and computers [14]. The choice of the communication protocol will depend on the range of the interlinked objects and the configuration state (i.e. passive or active). Communication protocols that can be used in LoTs include WiFi, Bluetooth, IEEE 802.15.4, RFID and LTE-Advanced.

\subsubsection{Computation}

Since the processing units and software applications represent the "brain" of the internet of things, these elements should have computational abilities in order to act according to parameter settings. The technology should be able to analyse data and understand appliance requests and come up with the most response.

\subsubsection{Services and semantics}

The success of the internet of things will depend on the ability of the connected objects to provide identity-related services, information aggregation services, collaborative-aware services and ubiquitous services [14]. This will facilitate object identification, data acquisition and reporting, decision 
making and response timeously within a given network of devices.

\section{LOAD MANAGEMENT TECHNIQUES IN POWER GRID}

Power utilities world over are faced with the problem of satisfying electric power demands. Meeting consumer demand for electric power is one of the major glitches faced by Power utilities [16]. Due to prohibitive costs in the addition of new generation capacity, Power utilities have designed programs and techniques in order to balance demand and supply within their generating capacity [17]; hence load management has come in as an important approach in providing electric power proportional in times of peak demands.

Load management can be seen as an effort both passive and active taken by Power utilities to reduce electric power consumption. It is concerned with the behaviours in the usage of electric power that affect energy efficiency during a congested period [18]. The passive approach ensures that the Power utility provides information or incentives that can persuade domestic consumers to minimise the usage of electricity, especially during peak periods. While on the other hand in the active approach, the Power utility takes full responsibility to control excess demands by using any of the following techniques [17]; (1) load shedding and restoring (2) power wheeling.

Load shedding and restoring is the process of interrupting consumer loads supplied under the same feeder or substation if they exceed load factor or are anticipated to do so [19]. In order to avoid continuous interruptions, the electric power is restored when the consumers are apparent that they are using other energy sources or industry demand has decreased significantly. Power utilities may use a pre-planned program in which load shedding is done according to timings where consumers are perceived to be requiring more electric power. Or they may use auto-programmed procedures such as the Breaker interlock, under frequency relay or Programmable Logic Controller (PLC).

In contrast, the concept of power wheeling is described by [20] as the process of transferring banked or reserved electric power from one substation to the other in order to cater for excess demand. This ensures that the substations support each other to avoid load shedding. However, for this process to take place, the transmission lines need to be interlinked with technology that has shifting capabilities.

\subsection{Load Management in Zimbabwe Electric Power Grid}

The two major approaches used by the Power utility in Zimbabwe are load shifting and load shedding. The first approach involves awareness campaigns that are disseminated to the consumers on national TVs, ZETDC revenue halls, power utility's website and newspapers in order to persuade them to reduce electric consumption. Since there are no incentives for low consumption of electric power, consumers only practice low consumption for their financial benefit; hence the approach has seen as to be passive in load management.

The second approach which is a Programmable Logic Controller Based Load Shedding is used in cases where load shifting and scheduled load shedding is not achieving the required load reduction [19]. In this approach, load shedding is instigated if the system frequency or load exceeds allowable maximum demand per feeder or substation. However, the major drawback in this approach is to determine the load reduction sequence. This makes it difficult to come up with the time of restoring electric power. As a result, the electric power is restored after a very long period.

\section{ROLE OF INTERNET OF THINGS IN LOAD MANAGEMENT}

The purpose of integrating the IoTs in the electric power grid is to enable the Power utility to turn off and on home appliances that have the potential of increasing the demand during peak periods [21]. This gives the Power utility the ability to interact with consumer appliances and actively control them. Using this technology, the Power utility can control the timings required by the appliance to connect to the electric power grid. This requires that the home appliances should be switched on/off using smart plugs. The technology should identify appliances that need to connect to the power grid system. The data acquired from the consumer side can be communicated to the control centre and back to the consumer. The IoTs is required to acquire connection data on the appliances that are perceived to be using high voltage. The IoTs is also needed to establish seamless and effective communication between context-aware sensors and the smart meter installed at the user site for automatic switching of the devices on/off based on load patterns [22].

\section{CONCEPTUAL MODEL OF IOTS IN LOAD MANAGEMENT}

This conceptual model was developed from the research gap found from the literature and the problem faced by the Power utility in Zimbabwe in balancing demand and supply. The model will ensure that electricity is available for basic and essential use in times of increased demand. The idea is modelled around continuous monitoring of the feeder or substation, activating the automatic control and carrying out load allocation procedure based on priority settings, connection, and re-connection timings. This will prevent the system from entering into and load shedding state. The concept further demands that all appliances connected to the electric power grid using smart plugs as shown in Figure 2. The system should utilise the power utility's own communication channel which is the PLC. 


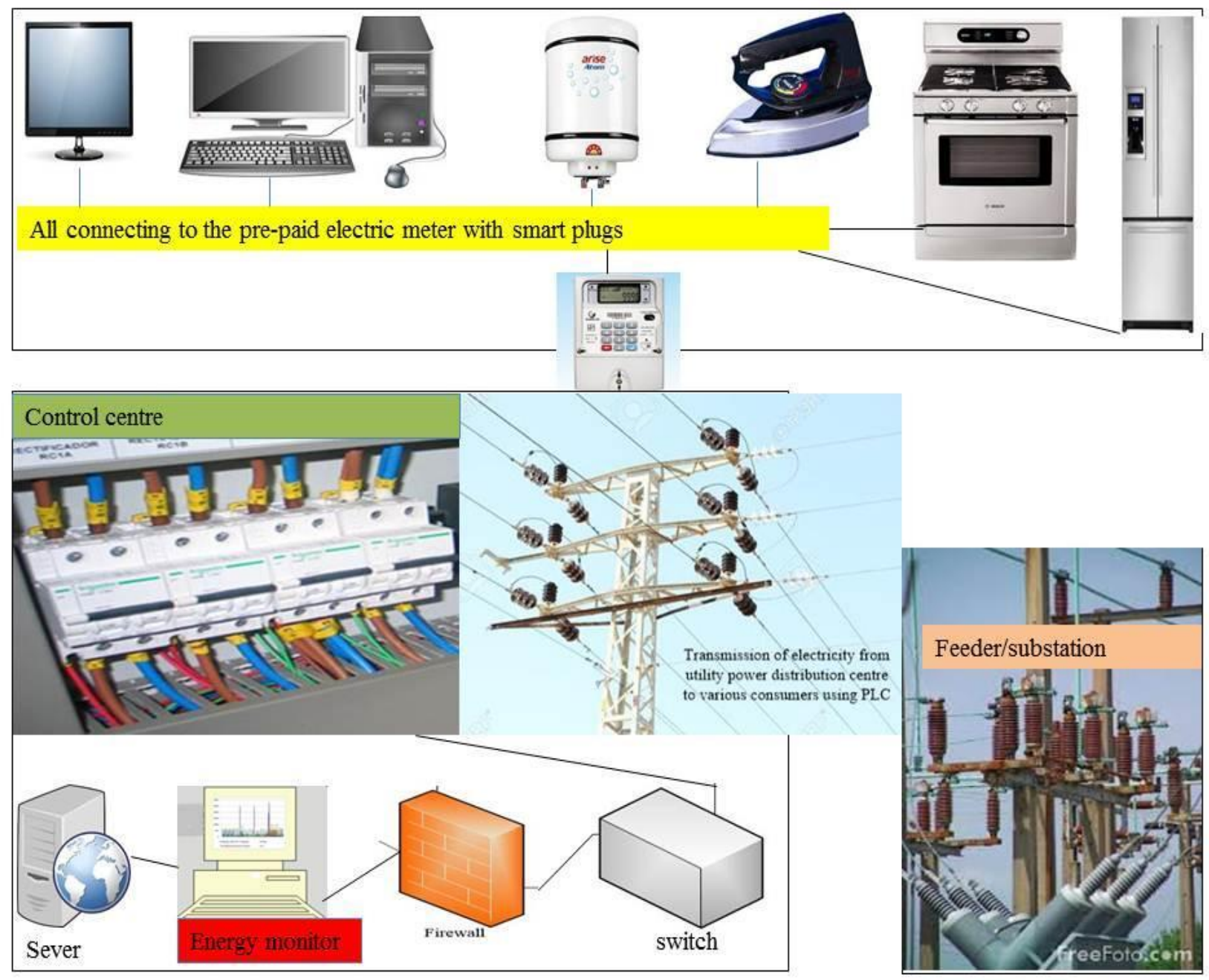

Fig 2: Conceptual model for load management: Own construction

\subsection{Algorithm for Load Management STEP 1: START}

STEP 2: Continuously monitor electric power demand from the feeder/ substation. IF Electric power demand is greater than $90 \%$ of the maximum allocated

THEN Activate automatic control and start demand allocation procedure ELSE continue with normal connectivity procedure

\section{STEP 3: STOP}

\subsection{Algorithm for Demand Allocation Procedure}

\section{STEP 1: START}

Set minimum and maximum connectivity time for low priority appliances,

Set re-connection time

STEP 2: Activate automatic control AND start sending warning signals to switch off low priority appliances.

STEP 3: Check priority settings of the new request from the database.

IF priority is high AND electric power demanded + current demand is $<$ maximum allocated

THEN
Permit appliance connectivity

ELSE

Halt connectivity AND automatically switch off all low priority appliances exceeded maximum connectivity time.

STEP 4: Connect halted requests IF the condition satisfies STEP 3.

STEP 5: IF condition on STEP 3 is not satisfied, disconnect other low priority appliances exceeded maximum connectivity time AND go to Step 4.

STEP 6: Check electric power demand level from the feeder/substation.

IF demand level is less than $90 \%$ of the maximum allocated THEN

De-activate automatic control

STEP 7: STOP allocation procedure

\subsection{Performance Evaluation}

Our proposed scheme was implemented in $\mathrm{C}++$, a high level programming language. In the performance evalaution, we consider for illustrative purposes, our algorithm is generalised into a 0-1 knapsack algorithm which is applied to the system of appliances (Figure 2) and their associated power 
consumptions as shown in Figure 3. For the sake of simplicity we consider only 4 appliances, but more can be included for larger systems. Furthermore we leverage on the works of [23] for a guide on consumption and priority aspects while deliberately ignoring the pricing regimes.

With regards to figure 3 , which is a bar graph, showing the appliances and their power consumption, it is apparent that devices 3 and 4 are the most likely heavy consumers of power. To this end, it is not surprising that at some instances given some demands the likely candidates to be connected are the devices 3 and 4 as shown in Figure 3. Clearly, appliance 4 is the heaviest consumer of power.

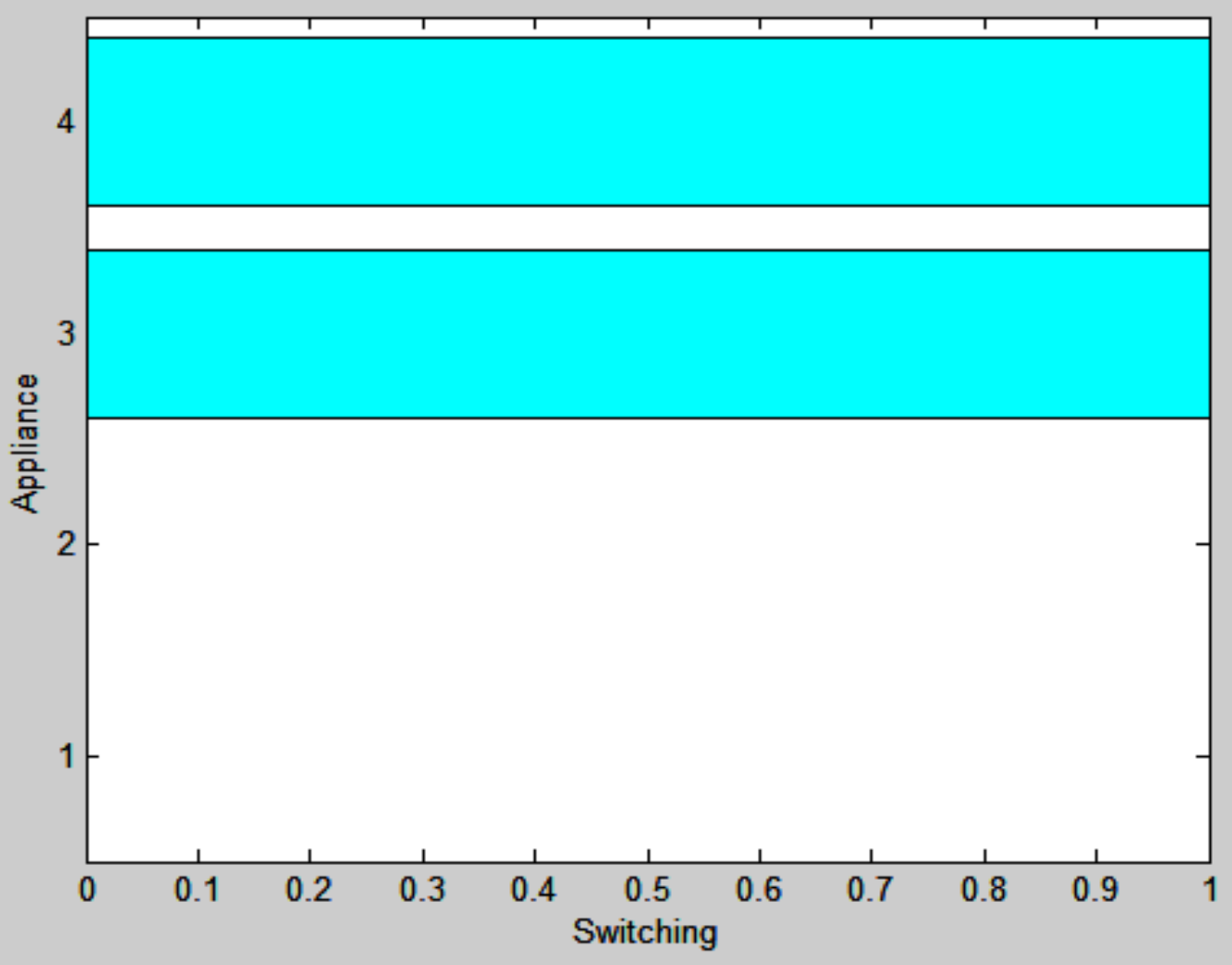

Fig 3: Switchable appliances 


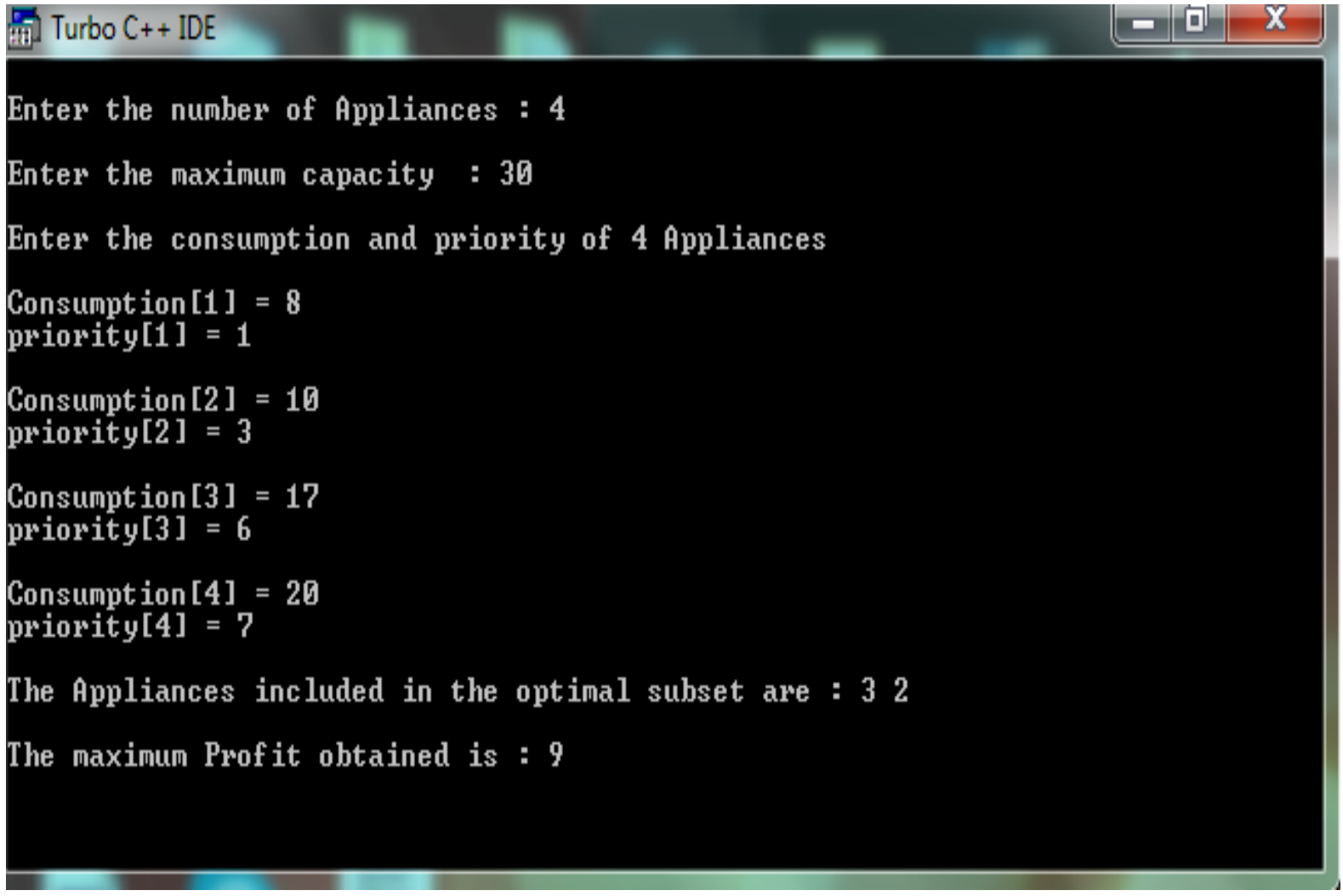

Fig 3b: Algorithmic testing

Figure $3 b$ shows the two appliances 3 and 4 as switchable based on the priority and consumption levels. Clearly, the bar graph lengths are the same for appliances 3 and 4 which are indicative of them being allowed to switch on under the given constraints. This is also confirmed by Figure $3 \mathrm{c}$ which shows the four appliances and the benefit of 9 referred to as profit in this context.

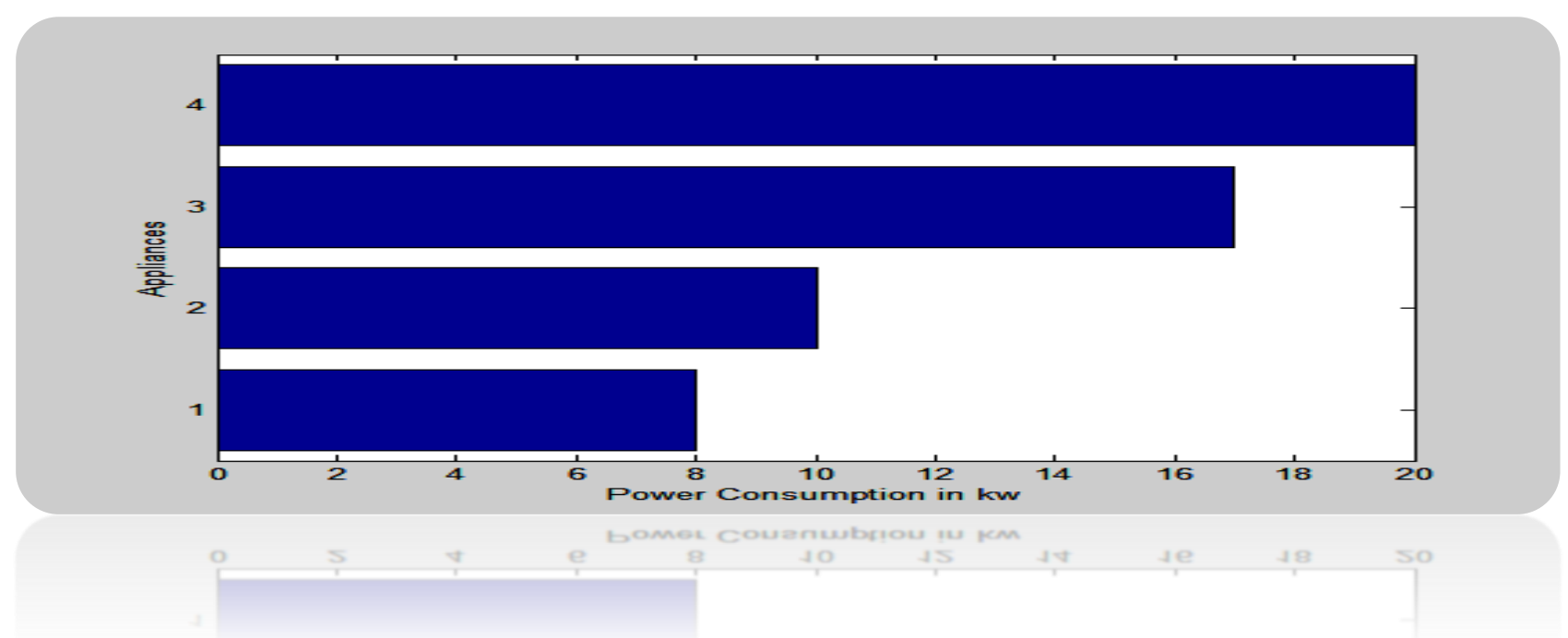

Fig 3c: Power Consumption of the appliances

However, in Figure 4, the demand for the connection is varied against the user's perception levels. Notably, as the demand for connection in Figure 4 increases, the user's perception priority levels increase steadily up to a demand of $35 \mathrm{kw}$.
Beyond this level of demand, there is again some significant increase till about $40 \mathrm{kw}$. Any demand beyond this appears not to change much in terms of the user's levels of priority 


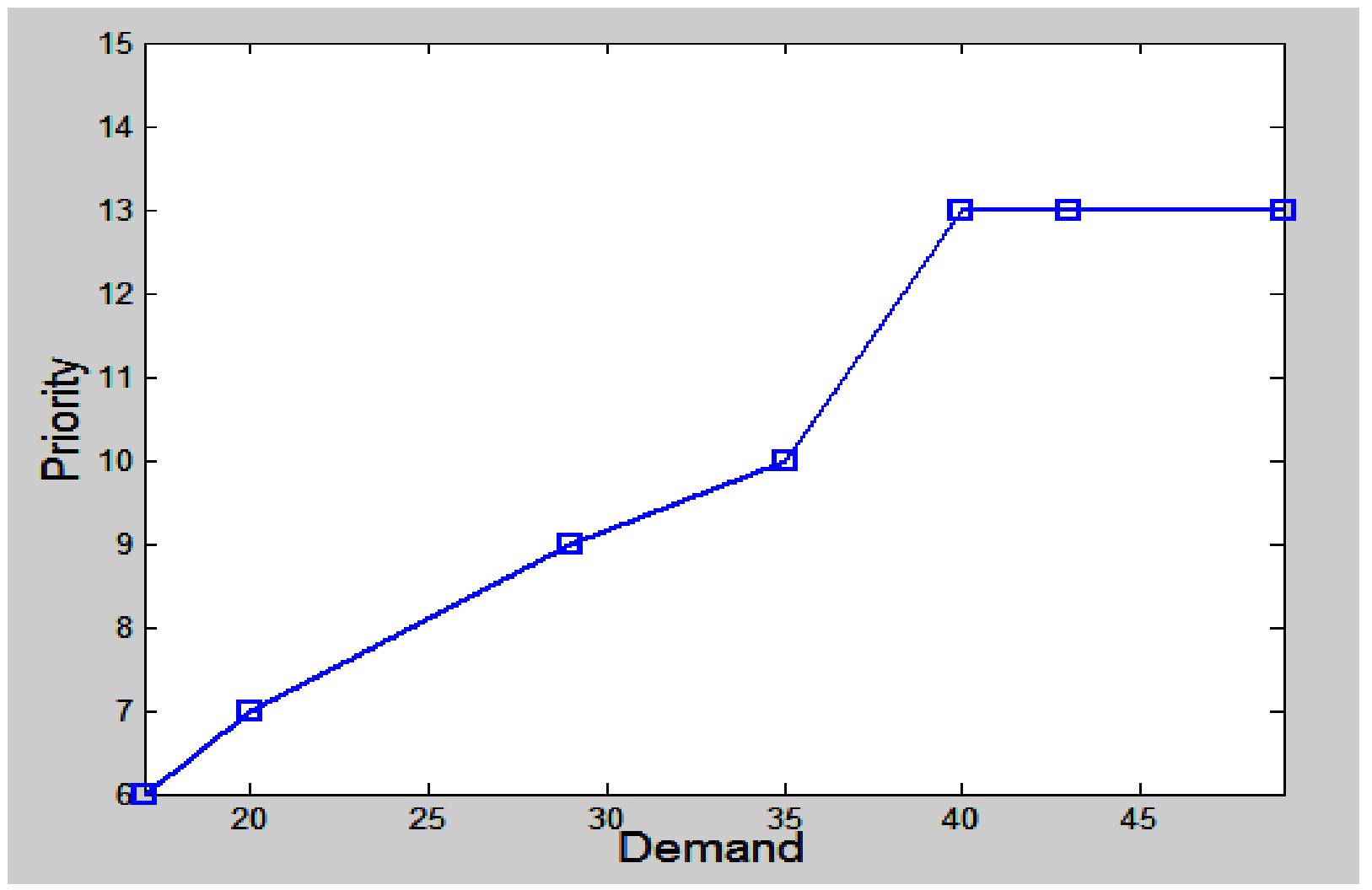

Fig 4: Priority and Demand Dependence

\section{CONCLUSION}

The dependence of demand and consumer priority perception is an interesting phenomenon in load management. This relationship in the context of our work may perhaps be attributed to an array of other factors such as pricing and income levels. However, Power utilities need to engage in more active techniques for load management. This is because human behaviour may lack the culture of conservation; the Power utility is required to come up with strong intervention strategies in achieving load management. Therefore, to cope with the demand of the power consumptions, power utilities need to embrace techniques that can balance the supply of electricity on the entire network. These techniques should allow the Power utility to interact with customer's load either direct or indirect. As consequence, this will ensure that electricity is available for basic and essential use in times of increased demand.

After the design, a prototype of the system was produced and tested; future work needs to extend the scope of the study by implementing the algorithm in a real world power grid network.

\section{REFERENCES}

[1] A. Shinn, K. Nakatani, and W. Rodriguez, "Analyzing the Role of the Intenet-of-Things in Business and Technologically-Smart Cities," vol. 6, no. 4, pp. 149$158,2017$.

[2] J. M. Mudumbe, "The internet of things for a smart South African grid architecture," no. 2014, pp. 95-107.

[3] R. Miceli, "Energy Management and Smart Grids," pp. 2262-2290, 2013.

[4] F. Aloul, A. R. Al-ali, R. Al-dalky, and M. Al-mardini, "Smart Grid Security: Threats, Vulnerabilities and
Solutions," Smart Grid Clean Energy Smart, no. 971, pp. $1-6,2012$.

[5] D. Volk, "Electricity Networks: Infrastructure and Operations Too complex for a resource ?," 2013.

[6] D. Newbery and A. Eberhard, "South African Network Infrastructure Review : Updated 2008 A paper written for National Treasury and the Department of Public Enterprises Government of South Africa," 2008.

[7] T. Foley et al., Renewables 2015 global status report. 2015.

[8] F. Mattern and C. Floerkemeier, "From the Internet of Computers to the Internet of Things."

[9] D. Evans, "The Internet of Things How the Next Evolution of the Internet The Internet of Things How the Next Evolution of the Internet Is Changing Everything," no. April, 2011.

[10] A. Crapo, R. Piasecki, D. Street, S. Francisco, and X. Wang, "The Smart Grid as a Semantically Enabled Internet of Things Grid-Interop Forum 2011,” 2011.

[11] S. Ramakrishnan, "WoT ( Web of Things ) for Energy Management in a Smart Grid-Connected Home," vol. 10, 2013.

[12] R. N. Calheiros, E. Alexandre, A. B. Do Carmo, C. A. F. De Rose, and R. Buyya, "Towards self-managed adaptive emulation of grid environments," Proc. - IEEE Symp. Comput. Commun., pp. 818-823, 2009.

[13] G. Misra, V. Kumar, A. Agarwal, and K. Agarwal, "Internet of Things ( IoT ) - A Technological Analysis and Survey on Vision, Concepts , Challenges , Innovation Directions, Technologies , and Applications ( 
An Upcoming or Future Generation Computer Communication System Technology )," vol. 4, no. 1, pp. 23-32, 2016.

[14] A. Al-fuqaha, S. Member, M. Guizani, M. Mohammadi, and S. Member, "Internet of Things: A Survey on Enabling," vol. 17, no. 4, pp. 2347-2376, 2015.

[15] C. Alcaraz, P. Najera, J. Lopez, and R. Roman, "Wireless Sensor Networks and the Internet of Things: Do We Need a Complete Integration ?," 2010.

[16] M. M. Eissa, S. M. Wasfy, and M. M. Sallam, "Load Management System Using Intelligent Monitoring and Control System for Commercial and Industrial Sectors," pp. 3-18, 2012.

[17] S. Ashok and R. Banerjee, "Load-management applications for the industrial sector."

[18] H. Svahnstr, "Demand Side Management in Smart Grids.'
[19] G. Shahgholian and M. E. Salary, "Effect of Load Shedding Strategy on Interconnected Power Systems Stability When a Blackout Occurs," vol. 4, no. 2, pp. 212-217, 2012

[20] J. Heeter, R. Vora, S. Mathur, N. Renewable, and P. Madrigal, "Wheeling and Banking Renewable Energy Wheeling and Banking Strategies for Optimal Renewable Energy Deployment: International Experiences," no. March, 2016.

[21] S. Barker, A. Mishra, D. Irwin, P. Shenoy, and J. Albrecht, "SmartCap: Flattening Peak Electricity Demand in Smart Homes."

[22] A. Reinhardt et al., "SmartMeter . KOM : A Low-cost Wireless Sensor for Distributed Power Metering," no. October, 2011.

[23] Rasheed et al., Energy optimization in smart homes using customer preference and dynamic pricing, 2016. 